\title{
Relatively lifting modules
}

\author{
Septimiu Crivei * \\ Faculty of Mathematics and Computer Science \\ "Babeş-Bolyai" University \\ 400084 Cluj-Napoca, Romania \\ E-mail: crivei@math.ubbcluj.ro
}

\begin{abstract}
We consider a generalization of lifting modules relative to a class $\mathcal{A}$ of modules and a proper class $\mathbb{E}$ of short exact sequences of modules. These modules will be called $\mathbb{E}$ - $\mathcal{A}$-lifting. We establish characterizations of modules with the property that every direct sum of copies of them is $\mathbb{E}$ - $\mathcal{A}$-lifting.
\end{abstract}

2000 Mathematics Subject Classification: 16S90, 16D80.

Keywords: Proper class, torsion theory, lifting module.

Received 25 August 2007

Revised 29 June 2008

Communicated by Nanqing Ding

\section{Introduction}

Let $\mathcal{A}$ be a class of modules closed under isomorphisms and containing the zero module. Al-Khazzi and Smith studied in [1] the class $d^{*} \mathcal{A}$ consisting of modules $A$ with the property that every submodule $B$ of $A$ contains a direct summand $C$ of $A$ such that $B / C \in \mathcal{A}$. The main motivation for their study was to offer a general setting for decomposing certain modules into a direct sum of a module in $\mathcal{A}$ and some other module. The modules in the class $d^{*} \mathcal{A}$ may also be seen as relative versions of the extensively investigated lifting modules (e.g., see [3]), that is, modules $A$ such that every submodule $B$ of $A$ contains a direct summand $C$ of $A$ such that $B / C$ is superfluous in $A / C$. Lifting modules have been generalized in [4] to $\mathbb{E}$-lifting modules by using instead of direct summands (i.e. splitting short exact sequences) elements of a proper class $\mathbb{E}$ of short exact sequences in the sense of Buchsbaum [2] or Mishina and Skornjakov [7].

In the present paper we put together the ideas from [1] and [4] in order to generalize the class $d^{*} \mathcal{A}$ by using such proper classes. The members of this new class of modules will be called $\mathbb{E}$ - $\mathcal{A}$-lifting modules. They generalize lifting modules, but also $\mathbb{E}$-lifting modules, since every $\mathbb{E}$-lifting module is $\mathbb{E}$ - $\mathcal{S}$-lifting, where $\mathcal{S}$ is the class of small modules. We also consider a specialization of this notion, called strongly $\mathbb{E}$ - $\mathcal{A}$-lifting module. We see the class $\mathcal{A}$ as a cogenerating class for a torsion theory $\tau$ in the category $\sigma[M]$ and we establish characterizations of $\Sigma$-(strongly) $\mathbb{E}$ - $\mathcal{A}$-lifting modules, that is, modules for which every direct sum of copies is (strongly) $\mathbb{E}$ - $\mathcal{A}$-lifting. As a consequence, we deduce that

*The author acknowledges the support of the grant CEEX-ET 19/2005. 
if a module $M$ is $\Sigma$ - $\mathcal{A}$-lifting, then every submodule of a direct sum of copies of $M$ is a direct sum of a module in $\mathcal{A}$ and a module in the class $\operatorname{Add}(M)$ of direct summands of direct sums of copies of $M$. Finally, for a $\Sigma$-E-lifting module $M$, we show that the property that a module belongs to a class generalizing the class $\operatorname{Add}(M)$ is lifted by certain epimorphisms.

Throughout $R$ is an associative ring with non-zero identity and all modules are unital right $R$-modules. By a class of modules we mean a class of modules closed under isomorphisms and containing the zero module. Throughout $M$ will be a module and $\mathcal{A}$ a class of modules in the category $\operatorname{Mod}-R$ of right $R$ modules. As usual, $M$ is said to be $\Sigma$-P $\left(\right.$ respectively $\prod-\mathcal{P}$ ) if every direct sum (respectively direct product) of copies of $M$ has the property $\mathcal{P}$. Denote by $\sigma[M]$ the full subcategory of Mod- $R$ whose objects are submodules of $M$-generated modules. By $\tau$ we denote a (not necessarily hereditary) torsion theory in $\sigma[M]$ and by $t(A)$ we denote the torsion submodule of a module $A$. Let $\mathcal{X}$ be any class of modules and $A$ be a module. Then $f \in \operatorname{Hom}(A, X)$, with $X \in \mathcal{X}$, is called an $\mathcal{X}$-preenvelope of $A$ if the induced abelian group homomorphism $\operatorname{Hom}\left(X, X^{\prime}\right) \rightarrow \operatorname{Hom}\left(A, X^{\prime}\right)$ is surjective for every $X^{\prime} \in \mathcal{X}$.

For further terminology concerning lifting modules and torsion theories the reader is referred to [3] and [10].

\section{Relatively coclosed modules}

Let us give the following definitions and some basic related properties.

Definition 2.1. (i) A submodule $C$ of a module $A$ is called $\mathcal{A}$-dense in $A$ if $A / C \in \mathcal{A}$.

(ii) A module $C$ is called $\mathcal{A}$-coclosed if $C / C^{\prime} \notin \mathcal{A}$ for every $C^{\prime}<C$.

Definition 2.2. Let $A$ be a module. A submodule $C$ of $A$ is called an $\mathcal{A}$ coclosure of $A$ if $C$ is an $\mathcal{A}$-dense submodule of $A$ and $C$ is $\mathcal{A}$-coclosed.

Lemma 2.3. Let $\mathcal{A}$ be closed under submodules and $C$ be a module. Then $C$ is $\mathcal{A}$-coclosed if and only if $\operatorname{Hom}(C, Y)=0$ for every $Y \in \mathcal{A}$.

Proof. Suppose that $C$ is $\mathcal{A}$-coclosed. Let $Y \in \mathcal{A}$ and $f \in \operatorname{Hom}(C, Y)$. Since $\operatorname{Im} f \subseteq Y \in \mathcal{A}$ and $C / \operatorname{Ker} f \cong \operatorname{Im} f$, we must have $\operatorname{Ker} f=C$, because otherwise $C$ is not $\mathcal{A}$-coclosed. Hence $f=0$, and so $\operatorname{Hom}(C, Y)=0$. Conversely, suppose that $\operatorname{Hom}(C, Y)=0$ for every $Y \in \mathcal{A}$ and let $C^{\prime}<C$. Then $\operatorname{Hom}\left(C, C / C^{\prime}\right) \neq 0$, hence $C / C^{\prime} \notin \mathcal{A}$. Thus $C$ is $\mathcal{A}$-coclosed.

The following well known technical result on torsion theories will be useful.

Lemma 2.4. Let $\mathcal{A} \subseteq \sigma[M]$ be closed under submodules and $\tau=(\mathcal{T}, \mathcal{F})$ be cogenerated by $\mathcal{A}$. Then $\mathcal{F}=\left\{N \mid \forall 0 \neq L \leq N, \exists L^{\prime}<L: L / L^{\prime} \in \mathcal{A}\right\}$.

Now we see $\mathcal{A}$ as a cogenerating class of $\tau$.

Lemma 2.5. Let $\tau$ be cogenerated by $\mathcal{A} \subseteq \sigma[M]$ and let $C$ be a module.

(i) If $C$ is $\tau$-torsion, then $C$ is $\mathcal{A}$-coclosed.

(ii) If $\mathcal{A}$ is closed under submodules and $C$ is $\mathcal{A}$-coclosed, then $C$ is $\tau$-torsion. 
Proof. (i) Clear.

(ii) Let $D=t(C)$. If $D \neq C$, then by Lemma 2.4 the $\tau$-torsionfree module $C / D$ has a proper submodule $C^{\prime} / D$ such that $C / C^{\prime} \cong(C / D) /\left(C^{\prime} / D\right) \in \mathcal{A}$, contradicting the fact that $C$ is $\mathcal{A}$-coclosed. Hence $D=C$, so that $C$ is $\tau$ torsion.

Corollary 2.6. Let $\mathcal{A} \subseteq \sigma[M]$ be the torsionfree class of $\tau, C$ be a module and $B$ be a submodules of $C$. Then:

(i) $C$ is $\mathcal{A}$-coclosed if and only if it is $\tau$-torsion.

(ii) $B$ is an $\mathcal{A}$-coclosure of $C$ if and only if $C$ is $\tau$-torsion and $C / B$ is $\tau$-torsionfree.

For the sake of brevity, let us say that a module $M$ has the $\mathcal{A}$-coclosure property if every submodule of $M$ has an $\mathcal{A}$-coclosure. In the following example we see that there are modules with the $\mathcal{A}$-coclosure property, but also without it.

Example 2.7. (i) Recall that a module $A \in \sigma[M]$ is called $M$-rational if $\operatorname{Hom}(C, M)=0$ for every submodule $C$ of $A$ [3, p. 84]. Let $\operatorname{Cogen}(M)$ be the class of $M$-cogenerated modules, that is, the class of modules $K$ for which there exists a monomorphism from $K$ to some direct product $M^{I}$. Then by Lemma 2.3 it follows easily that a module $A \in \sigma[M]$ is $M$-rational if and only if every submodule of $A$ is Cogen $(M)$-coclosed. Moreover, clearly every $M$-rational module has the Cogen $(M)$-coclosure property.

(ii) Let $\mathcal{Z}$ be the class consisting of the zero modules and the simple modules. Also, let $B$ be a module with the radical consisting of a simple module, say $D$, which is also a maximal submodule of $B$ (and so $B$ is a module of composition length 2). We claim that $B$ does not have a $\mathcal{Z}$-coclosure. Suppose the contrary and denote by $C$ a $\mathcal{Z}$-coclosure of $B$. Then $B / C \in \mathcal{Z}$, hence $C$ could be either $B$ or $D$. But neither $B$ nor $D$ is $\mathcal{Z}$-coclosed, because we have $B / D \in \mathcal{Z}$ and $D \in \mathcal{Z}$. This is a contradiction, so the claim follows.

\section{Relatively lifting modules and proper classes}

Recall the definition of a proper class of short exact sequences (e.g., see [3, 10.1]).

Definition 3.1. Let $\mathbb{E}$ be a class of short exact sequences in Mod- $R$. If an exact sequence $0 \rightarrow K \stackrel{f}{\rightarrow} L \stackrel{g}{\rightarrow} N \rightarrow 0$ belongs to $\mathbb{E}$, then $f$ is called an $\mathbb{E}$-monomorphism and $g$ is called an $\mathbb{E}$-epimorphism. Also, $\operatorname{Im} f$ is called an $\mathbb{E}$-submodule of $L$ and $N$ is called an $\mathbb{E}$-homomorphic image of $L$.

The class $\mathbb{E}$ is called a proper class if it has the following properties:

$\mathrm{P} 1 . \mathbb{E}$ is closed under isomorphisms;

P2. $\mathbb{E}$ contains all splitting short exact sequences;

P3. the class of $\mathbb{E}$-monomorphisms is closed under composition;

if $f, f^{\prime}$ are monomorphisms and $f^{\prime} f$ is an $\mathbb{E}$-monomorphism, then $f$ is an $\mathbb{E}$-monomorphism;

P4. the class of $\mathbb{E}$-epimorphisms is closed under composition;

if $g, g^{\prime}$ are epimorphisms and $g g^{\prime}$ is an $\mathbb{E}$-epimorphism, then $g$ is an $\mathbb{E}$ epimorphism. 
Example 3.2. Some examples of proper classes are the following (e.g., see [3]):

(i) The class $\mathbb{E}_{s}$ of all splitting short exact sequences in Mod- $R$.

(ii) The class $\mathbb{E}^{\mathcal{X}}$ of all short exact sequences in Mod- $R$ on which the functor $\operatorname{Hom}(X,-)$ is exact for every $X \in \mathcal{X}$, where $\mathcal{X}$ is any class of modules in Mod- $R$. Its elements are called $\mathcal{X}$-pure exact sequences. For the class $\mathcal{X}=\mathcal{P}$ of finitely presented modules, one has the classical pure exact sequences.

Throughout, $\mathbb{E}$ will be a proper class of short exact sequences in Mod- $R$. We introduce the following definition.

Definition 3.3. A module $A$ is called $\mathbb{E}$ - $\mathcal{A}$-lifting if every submodule $B$ of $A$ contains an $\mathbb{E}$-submodule $C$ of $A$ such that $C$ is $\mathcal{A}$-dense in $B$.

For $\mathbb{E}=\mathbb{E}_{s}$, we call $\mathbb{E}_{s}-\mathcal{A}$-lifting modules simply $\mathcal{A}$-lifting. Note that the class of $\mathcal{A}$-lifting modules is exactly the class $d^{*} \mathcal{A}$ from the introduction.

Example 3.4. (i) Every semisimple module is $\mathbb{E}$ - $\mathcal{A}$-lifting.

(ii) Let $\mathcal{O}$ be the class of zero modules. Then a module is $\mathcal{O}$-lifting if and only if it is semisimple. Also, a module is $\mathbb{E}^{\mathcal{P}}$ - $\mathcal{O}$-lifting if and only if it is regular in the sense of [11, Chapter 37].

(iii) Recall that a module $A$ is called $\tau$-supplemented if every submodule $B$ of $A$ contains a direct summand $C$ of $A$ such that $B / C$ is $\tau$-torsion [6]. If $\mathcal{A}$ is the torsion class of $\tau$, then $\mathcal{A}$-lifting means $\tau$-supplemented.

(iv) Recall that a module $A \in \sigma[M]$ is called $M$-small if $A$ is superfluous in some module $A^{\prime} \in \sigma[M]$. Also, recall that a module $A$ is called $\mathbb{E}$-lifting if every submodule $B$ of $A$ contains an $\mathbb{E}$-submodule $C$ of $A$ such that $B / C$ is superfluous in $A / C$ [4]. If $A \in \sigma[M]$ is $\mathbb{E}$-lifting, then it is clearly $\mathbb{E}$ - $\mathcal{S}$-lifting, where $\mathcal{S}$ is the class of $M$-small modules. In particular, every lifting module is $\mathcal{S}$-lifting.

(v) Let $\tau$ be cogenerated by $\mathcal{A} \subseteq \sigma[M]$ and suppose that $\tau$ is cohereditary. If $A$ is $\tau$-torsion $\mathcal{A}$-lifting, then it is lifting. Indeed, if $B$ is a submodule of $A$, then it contains some direct summand $C$ of $A$ such that $B / C \in \mathcal{A}$, hence $B / C$ is $\tau$-torsionfree. We claim that $X=B / C$ is superfluous in $Y=A / C$. If $Z<Y$, then we have $Z+X \neq Y$, because otherwise the non-zero module $X /(Z \cap X)$ would be both $\tau$-torsion, being isomorphic to $Y / Z$, and $\tau$-torsionfree, because $\tau$ is cohereditary. This shows that $A$ is lifting.

Lemma 3.5. (i) Let $A$ be an $\mathbb{E}$-A-lifting module. Then every $\mathcal{A}$-coclosed submodule of $A$ is an $\mathbb{E}$-submodule.

(ii) Let $A$ be a module with the $\mathcal{A}$-coclosure property such that every $\mathcal{A}$ coclosed submodule of $A$ is an $\mathbb{E}$-submodule. Then $A$ is $\mathbb{E}$ - $\mathcal{A}$-lifting.

(iii) The class of $\mathbb{E}$ - $\mathcal{A}$-lifting modules is closed under submodules.

Proof. (i) Let $B$ be an $\mathcal{A}$-coclosed submodule of $A$. Then $B$ contains an $\mathbb{E}$ submodule $C$ of $A$ such that $B / C \in \mathcal{A}$. Then $B=C$, because otherwise we would have $B / C \notin \mathcal{A}$ since $B$ is $\mathcal{A}$-coclosed. Hence $B$ is an $\mathbb{E}$-submodule of $A$.

(ii) Let $B$ be a submodule of $A$ and $C$ be an $\mathcal{A}$-coclosure of $B$. Then $B / C \in \mathcal{A}$ and $C$ is $\mathcal{A}$-coclosed, so that $C$ is an $\mathbb{E}$-submodule. Hence $A$ is $\mathbb{E}$ - $\mathcal{A}$-lifting.

(iii) Let $A$ be an $\mathbb{E}$ - $\mathcal{A}$-lifting module and $D$ be a submodule of $A$. Let $B$ be a submodule of $D$. Then $B$ contains an $\mathbb{E}$-submodule $C$ of $A$ such that $B / C \in \mathcal{A}$. Then $C$ is an $\mathbb{E}$-submodule of $D$, showing that $D$ is $\mathbb{E}$ - $\mathcal{A}$-lifting. 


\section{$4 \quad \Sigma$-E- $\mathcal{A}$-lifting modules}

Following [4], we denote by $\mathbb{E} \operatorname{Prod}(M)\left(\right.$ respectively $\left.\mathbb{E} \operatorname{Prod}^{\prime}(M)\right)$ the class of modules $K$ for which there is an $\mathbb{E}$-monomorphism from $K$ to some direct product $M^{I}$ (respectively direct sum $M^{(I)}$ ) of copies of $M$ and by $\operatorname{Cogen}(M)$ (respectively $\left.\operatorname{Cogen}^{\prime}(M)\right)$ the class of modules $K$ for which there exists a monomorphism from $K$ to some $M^{I}$ (respectively $M^{(I)}$ ). For instance, $\mathbb{E}_{s} \operatorname{Prod}(M)$ (respectively $\mathbb{E}_{s} \operatorname{Prod}^{\prime}(M)$ ) is the class $\operatorname{Prod}(M)$ (respectively $\operatorname{Add}(M)$ ) of direct summands of direct products (respectively direct sums) of copies of $M$.

We need the following lemma, whose proof is straightforward taking into account that the composition of two $\mathbb{E}$-monomorphisms is again an $\mathbb{E}$-monomorphism.

Lemma 4.1. [4, Lemma 3.1] The classes $\mathbb{E} \operatorname{Prod}(M)$ and $\mathbb{E} \operatorname{Prod}^{\prime}(M)$ are both closed under $\mathbb{E}$-submodules.

Recall that a module is called direct injective if for every direct summand $X$ of $M$, every monomorphism $X \rightarrow M$ splits (for instance, see [5, 2.11]). In our context, we need a generalization of direct injectivity with respect to proper classes, which was considered in [4].

Definition 4.2. A module $M$ is called $\mathbb{E}$-direct injective if, for every $\mathbb{E}$-submodule $X$ of $M$, every monomorphism $X \rightarrow M$ is an $\mathbb{E}$-monomorphism.

$\Sigma$-E-direct injective modules may be characterized as follows. We sketch a proof for the reader's convenience.

Lemma 4.3. [4, Lemma 4.6] A module $M$ is $\Sigma$-E-direct injective if and only if for every $U \in \operatorname{Cogen}^{\prime}(M)$ and every $V \in \mathbb{E} \operatorname{Prod}^{\prime}(M)$, every monomorphism $V \rightarrow U$ is an $\mathbb{E}$-monomorphism.

Proof. Suppose first that $M$ is $\Sigma$-E-direct injective. Let $U \in \operatorname{Cogen}^{\prime}(M)$ and $V \in \mathbb{E} \operatorname{Prod}^{\prime}(M)$ and let $f: V \rightarrow U$ be a monomorphism. Then there exist a monomorphism $g: U \rightarrow M^{(I)}$ and an $\mathbb{E}$-monomorphism $h: V \rightarrow M^{(J)}$. Let us consider the monomorphism igf $: V \rightarrow M^{(I)} \oplus M^{(J)}$, where $i: M^{(I)} \rightarrow M^{(I)} \oplus$ $M^{(J)}$ is the inclusion monomorphism. Since we may see $V$ as an $\mathbb{E}$-submodule of $M^{(I)} \oplus M^{(J)}$ and $M$ is $\Sigma$ - $\mathbb{E}$-direct injective, igf is an $\mathbb{E}$-monomorphism, hence $f$ has to be an $\mathbb{E}$-monomorphism. The converse is clear.

Now we can establish our main result on $\Sigma$ - $\mathbb{E}$ - $\mathcal{A}$-lifting modules.

Theorem 4.4. Let $\tau$ be cogenerated by $\mathcal{A} \subseteq \sigma[M]$. Consider the following statements:

(a) $M$ is $\Sigma$-E-A-lifting;

(b) Every module in $\operatorname{Add}(M)$ is $\mathbb{E}$-A-lifting;

(c) Every $K \in \operatorname{Cogen}^{\prime}(M)$ has an $\mathbb{E}$-homomorphic image $K / Y \in \mathcal{A}$ such that $Y \in \mathbb{E} \operatorname{Prod}^{\prime}(M)$

(d) Every $\tau$-torsion module in $\operatorname{Cogen}^{\prime}(M)$ is in $\mathbb{E} \operatorname{Prod}^{\prime}(M)$;

(e) Every $\tau$-torsion module in $\operatorname{Cogen}^{\prime}(M)$ is $\mathbb{E}$-A-lifting.

Then the following implications hold: 
1. For every module $M,(a) \Leftrightarrow(b) \Rightarrow(c) \Rightarrow(d)$.

2. If $M$ is $\Sigma$-E-direct injective, then $(c) \Rightarrow(a)$.

3. If $M$ is $\Sigma$-E-direct injective, has the $\Sigma$-A-coclosure property, and $\mathcal{A}$ is closed under submodules, then $(d) \Rightarrow(e)$.

4. If $M$ is $\tau$-torsion, then $(e) \Rightarrow(a)$.

Proof. (1) (a) $\Leftrightarrow$ (b) Suppose that $M$ is $\Sigma$-E- $\mathcal{A}$-lifting and let $N \in \operatorname{Add}(M)$. Then there is a monomorphism $N \rightarrow M^{(I)}$. Now by Lemma 3.5 it follows that $N$ is $\mathbb{E}$ - $\mathcal{A}$-lifting. The converse is obvious.

(b) $\Rightarrow$ (c) Let $K \in \operatorname{Cogen}^{\prime}(M)$ and take a monomorphism $f: K \rightarrow M^{(I)}$. Since $M^{(I)}$ is $\mathbb{E}$ - $\mathcal{A}$-lifting, $f(K)$ contains an $\mathbb{E}$-submodule $L$ such that $f(K) / L \in$ $\mathcal{A}$. If $Y=f^{-1}(L)$, then it follows that $K / Y \in \mathcal{A}, Y \in \mathbb{E} \operatorname{Prod}^{\prime}(M)$ and $Y$ is an $\mathbb{E}$-submodule of $K$.

(c) $\Rightarrow$ (d) Clear.

(2) Assume that $M$ is $\Sigma$-E-direct injective.

(c) $\Rightarrow$ (a) Let $I$ be a set and $K$ be a submodule of $M^{(I)}$. Then by hypothesis $K$ has an $\mathbb{E}$-homomorphic image $K / Y \in \mathcal{A}$ such that $Y \in \mathbb{E} \operatorname{Prod}^{\prime}(M)$. Then by Lemma 4.3 , the inclusion monomorphism $Y \rightarrow M^{(I)}$ is an $\mathbb{E}$-monomorphism, hence $Y$ is an $\mathbb{E}$-submodule of $M^{(I)}$. Thus $M^{(I)}$ is $\mathbb{E}$ - $\mathcal{A}$-lifting.

(3) Assume that $M$ is $\Sigma$-E-direct injective, has the $\Sigma$ - $\mathcal{A}$-coclosure property, and $\mathcal{A}$ is closed under submodules.

$(\mathrm{d}) \Rightarrow(\mathrm{e})$ Let $K$ be a $\tau$-torsion module in $\operatorname{Cogen}^{\prime}(M)$ and consider a monomorphism $f: K \rightarrow M^{(I)}$. Let $L$ be a proper submodule of $K$. Then $f(L)$ has an $\mathcal{A}$-coclosure, say $C$. It follows that $C$ is $\tau$-torsion by Lemma 2.5. Since $C \in \operatorname{Cogen}^{\prime}(M)$, we have $C \in \mathbb{E} \operatorname{Prod}^{\prime}(M)$ by hypothesis. Now by Lemma 4.3 the inclusion $C \rightarrow f(K)$ is an $\mathbb{E}$-monomorphism. Then the inclusion $f^{-1}(C) \rightarrow K$ is an $\mathbb{E}$-monomorphism. Since $f^{-1}(C)$ is $\mathcal{A}$-coclosed, it follows that $K$ is $\mathbb{E}-\mathcal{A}$ lifting.

(4) Assume that $M$ is $\tau$-torsion.

(e) $\Rightarrow$ (a) If $M$ is $\tau$-torsion, then every $M^{(I)}$ is $\tau$-torsion, hence $\mathbb{E}$ - $\mathcal{A}$-lifting.

For the proper class $\mathbb{E}=\mathbb{E}_{s}$ we obtain the following consequence.

Corollary 4.5. Let $\tau$ be cogenerated by $\mathcal{A} \subseteq \sigma[M]$. Consider the following statements:

(a) $M$ is $\Sigma$-A-lifting;

(b) Every module in $\operatorname{Add}(M)$ is $\mathcal{A}$-lifting;

(c) Every module in $\operatorname{Cogen}^{\prime}(M)$ is a direct sum of a module in $\mathcal{A}$ and a module in $\operatorname{Add}(M)$;

(d) Every $\tau$-torsion module in $\operatorname{Cogen}^{\prime}(M)$ is in $\operatorname{Add}(M)$;

(e) Every $\tau$-torsion module in $\operatorname{Cogen}^{\prime}(M)$ is $\mathcal{A}$-lifting.

Then the following implications hold:

1. For every module $M,(a) \Leftrightarrow(b) \Rightarrow(c) \Rightarrow(d)$. 
2. If $M$ is $\Sigma$-direct injective, then (c) $\Rightarrow(a)$.

3. If $M$ is $\Sigma$-direct injective, has the $\Sigma$-A-coclosure property, and the class $\mathcal{A}$ is closed under submodules, then $(d) \Rightarrow(e)$.

4. If $M$ is $\tau$-torsion, then (e) $\Rightarrow(a)$.

Corollary 4.6. Let $\tau$ be the torsion theory in $\operatorname{Mod}-R$ cogenerated by $\mathcal{A}$. Consider the following statements:

(a) $R$ is right $\Sigma$-A-lifting;

(b) Every projective module is $\mathcal{A}$-lifting;

(c) Every submodule of a free module is a direct sum of a module in $\mathcal{A}$ and a projective module;

(d) Every $\tau$-torsion submodule of a free module is projective;

(e) Every $\tau$-torsion submodule of a free module is $\mathcal{A}$-lifting.

Then the following implications hold:

1. For any $R,(a) \Leftrightarrow(b) \Rightarrow(c) \Rightarrow(d)$.

2. If $R$ is $\Sigma$-direct injective, then (c) $\Rightarrow(a)$.

3. If $R$ is $\Sigma$-direct injective, has the $\Sigma$-A-coclosure property, and the class $\mathcal{A}$ is closed under submodules, then $(d) \Rightarrow(e)$.

4. If $R$ is $\tau$-torsion, then $(e) \Rightarrow(a)$.

One may further particularize Theorem 4.4 to some classes $\mathcal{A}$, of which the classes of $\tau$-supplemented or $M$-small modules (see Example 3.4) are of interest.

\section{$5 \quad \Sigma$-strongly $\mathbb{E}$ - $\mathcal{A}$-lifting modules}

Now let us consider a natural intermediate notion between those of $\mathcal{A}$-lifting module and $\mathbb{E}$ - $\mathcal{A}$-lifting module.

Definition 5.1. A module $M$ is called strongly $\mathbb{E}$ - $\mathcal{A}$-lifting if $M$ has the $\mathcal{A}$ coclosure property and the $\mathcal{A}$-coclosed submodules of $M$ coincide with its $\mathbb{E}$ submodules.

Lemma 5.2. Let $A$ be a strongly $\mathbb{E}$-A-lifting module and $D$ be an $\mathcal{A}$-coclosed submodule ( $\mathbb{E}$-submodule) of $A$. Then $D$ is strongly $\mathbb{E}$ - $\mathcal{A}$-lifting.

Proof. By Lemma 3.5, $D$ is $\mathbb{E}$ - $\mathcal{A}$-lifting. Since $A$ has the $\mathcal{A}$-coclosure property, then clearly so does any submodule of $A$. Finally, let $B$ be an $\mathbb{E}$-submodule of $D$. Since $D$ is an $\mathbb{E}$-submodule of $A, B$ is an $\mathbb{E}$-submodule of $A$, and so an $\mathcal{A}$-closed submodule of $A$. Therefore, $D$ is strongly $\mathbb{E}$ - $\mathcal{A}$-lifting.

In the following result we characterize $\Sigma$-strongly $\mathbb{E}$ - $\mathcal{A}$-lifting modules.

Theorem 5.3. Let $\tau$ be cogenerated by $\mathcal{A} \subseteq \sigma[M]$. Consider the following statements: 
(a) $M$ is $\Sigma$-strongly $\mathbb{E}$ - $\mathcal{A}$-lifting;

(b) Every module in $\operatorname{Add}(M)$ is strongly $\mathbb{E}$-A-lifting;

(c) Every module in $\mathbb{E} \operatorname{Prod}^{\prime}(M)$ is strongly $\mathbb{E}$-A-lifting;

(d) Every $\tau$-torsion module in $\operatorname{Cogen}^{\prime}(M)$ is strongly $\mathbb{E}$-A-lifting;

(e) $\mathbb{E} \operatorname{Prod}^{\prime}(M)$ consists of the $\tau$-torsion modules in $\operatorname{Cogen}^{\prime}(M)$.

Then the following implications hold:

1. For every module $M,(a) \Leftrightarrow(b) \Leftrightarrow(c)$.

2. If $\mathcal{A}$ is closed under submodules, then $(a) \Rightarrow(e)$ and $(a) \Rightarrow(d)$.

3. If $M$ is $\tau$-torsion, then $(d) \Rightarrow(a)$.

Proof. (1) (a) $\Rightarrow$ (c) Let $K \in \mathbb{E} \operatorname{Prod}^{\prime}(M)$. Then there is an $\mathbb{E}$-monomorphism $K \rightarrow M^{(I)}$. Now by Lemma $5.2, K$ is strongly $\mathbb{E}$ - $\mathcal{A}$-lifting.

(c) $\Rightarrow(\mathrm{b}) \Rightarrow(\mathrm{a})$ Clear.

(2) Assume that $\mathcal{A}$ is closed under submodules.

(a) $\Rightarrow\left(\right.$ e) By Theorem 4.4, every $\tau$-torsion module in $\operatorname{Cogen}^{\prime}(M)$ is in $\mathbb{E} \operatorname{Prod}^{\prime}(M)$. Conversely, let $K \in \mathbb{E} \operatorname{Prod}^{\prime}(M)$ and take some $\mathbb{E}$-monomorphism $g: K \rightarrow M^{(I)}$. Then $K$ is an $\mathbb{E}$-submodule of $M^{(I)}$, hence $\mathcal{A}$-coclosed in $M^{(I)}$. Now by Lemma 2.5 it follows that $K$ is $\tau$-torsion.

(a) $\Rightarrow$ (d) By (c), every module in $\mathbb{E} \operatorname{Prod}^{\prime}(M)$ is strongly $\mathbb{E}$ - $\mathcal{A}$-lifting. Then by (e) it follows that every $\tau$-torsion module in $\operatorname{Cogen}^{\prime}(M)$ is strongly $\mathbb{E}$ - $\mathcal{A}$-lifting.

(3) Assume that $M$ is $\tau$-torsion.

(d) $\Rightarrow$ (a) If $M$ is $\tau$-torsion, then every $M^{(I)}$ is $\tau$-torsion, hence strongly $\mathbb{E}$ $\mathcal{A}$-lifting.

Corollary 5.4. Let $\tau$ be cogenerated by $\mathcal{A} \subseteq \sigma[M]$. Consider the following statements:

(a) $M$ is $\Sigma$-strongly $\mathcal{A}$-lifting;

(b) Every module in $\operatorname{Add}(M)$ is strongly A-lifting;

(c) Every $\tau$-torsion module in $\operatorname{Cogen}^{\prime}(M)$ is strongly $\mathcal{A}$-lifting;

(d) $\operatorname{Add}(M)$ consists of the $\tau$-torsion modules in $\operatorname{Cogen}^{\prime}(M)$.

Then the following implications hold:

1. For every module $M,(a) \Leftrightarrow(b)$.

2. If $\mathcal{A}$ is closed under submodules, then $(a) \Rightarrow(d)$ and $(a) \Rightarrow(c)$.

3. If $M$ is $\tau$-torsion, then $(c) \Rightarrow(a)$.

Corollary 5.5. Let $\tau$ be the torsion theory in Mod- $R$ cogenerated by a class $\mathcal{A}$ closed under submodules and suppose that $R$ is $\tau$-torsion. Then the following are equivalent:

(a) $R$ is right $\Sigma$-strongly $\mathcal{A}$-lifting;

(b) Every projective module is strongly $\mathcal{A}$-lifting;

(c) Every submodule of a free module is strongly $\mathcal{A}$-lifting;

In this case, we also have:

(d) A module is projective if and only if it is a submodule of a free module. 


\section{$6 \quad \Sigma-\mathbb{E}$ - $\mathcal{A}$-lifting modules and relative preenvelopes}

An important result of Oshiro [8, Theorem II] says that if $R$ is right $\Sigma$-extending, then the class of projective modules is closed under essential extensions. Motivated by this, we establish in our case a result with dual flavor. Thus, for a $\Sigma-\mathbb{E}$ $\mathcal{A}$-lifting module $M$ and an epimorphism $Y \rightarrow Z$ we study when $Z \in \mathbb{E} \operatorname{Prod}^{\prime}(M)$ implies $Y \in \mathbb{E} \operatorname{Prod}^{\prime}(M)$.

The following condition on a module $M$ will be useful:

(*) For every proper submodules $B, C, D$ of $M$ with $D+B=M$ and $C$ $\mathcal{A}$-dense in $B$ we have $D+C=M$.

For instance, any hollow module clearly satisfies $(*)$.

We need the following technical lemma.

Lemma 6.1. Let $p: K \rightarrow M$ be a monomorphism such that $M$ is $\mathbb{E}$-A-lifting and $K$ satisfies (*). If there exists a proper submodule $D$ of $M$ such that $D+$ $\operatorname{Im} p=M$ and $p^{-1}(D) \in \mathcal{A}$, then $\operatorname{Im} p$ is an $\mathbb{E}$-submodule of $M$.

Proof. We may assume that $N=\operatorname{Im} p$ is a proper submodule of $M$. Since $M$ is $\mathbb{E}$ - $\mathcal{A}$-lifting, $N$ contains an $\mathbb{E}$-submodule $L$ of $M$ such that $N / L \in \mathcal{A}$. Since $D+N=M$, we have $D+L=M$, whence it follows easily that $p^{-1}(D)+$ $p^{-1}(L)=K$. This and the fact that $p^{-1}(D) \in \mathcal{A}$ implies by hypothesis that $p^{-1}(L)=p^{-1}(L)+0=K$, whence $N \subseteq L$. Thus $N=L$ is an $\mathbb{E}$-submodule of $M$.

Theorem 6.2. Let $M$ be $\Sigma$ - $\mathbb{E}$-A-lifting. If $j: Y \rightarrow Z$ is a non-zero epimorphism such that $\operatorname{Ker} j \in \mathcal{A}, Z \in \mathbb{E} \operatorname{Prod}^{\prime}(M), Y \in \operatorname{Cogen}^{\prime}(M)$ and $Y$ satisfies (*) and has an $\mathbb{E P r o d}^{\prime}(M)$-preenvelope, then $Y \in \mathbb{E P r o d}^{\prime}(M)$.

Proof. Let $p: Y \rightarrow E$ be an $\mathbb{E} \operatorname{Prod}^{\prime}(M)$-preenvelope of $Y$. Then $j$ factors through $p$, hence there is a homomorphism $q: E \rightarrow Z$ such that $q p=j$. Since there exists some $\mathbb{E}$-monomorphism $E \rightarrow M^{(I)}, E$ is $\mathbb{E}$ - $\mathcal{A}$-lifting by Lemma 3.5. Since $Y \in \operatorname{Cogen}^{\prime}(M)$, it follows that $p$ is a monomorphism. Then we have $\operatorname{Ker} q \neq E$ and $p^{-1}(\operatorname{Ker} q)=\operatorname{Ker} j \in \mathcal{A}$. It is easy to check that $\operatorname{Ker} q+\operatorname{Im} p=E$, whence $\operatorname{Im} p$ is an $\mathbb{E}$-submodule of $E$ by Lemma 6.1. Since $E \in \mathbb{E} \operatorname{Prod}^{\prime}(M)$, it follows by Lemma 4.1 that $Y \in \mathbb{E} \operatorname{Prod}^{\prime}(M)$.

Corollary 6.3. Let $M$ be $\Sigma$-E-A-lifting. If $j: Y \rightarrow Z$ is a non-zero epimorphism such that $\operatorname{Ker} j \in \mathcal{A}, Z \in \operatorname{Add}(M), Y \in \operatorname{Cogen}^{\prime}(M)$ and $Y$ satisfies (*) and has an $\operatorname{Add}(M)$-preenvelope, then $Y \in \operatorname{Add}(M)$.

Note that one may replace direct sums with direct products in Theorem 6.2 and obtain a similar result. Every module has an $\operatorname{Add}(M)$-preenvelope if and only if $\operatorname{Add}(M)$ is closed under products, and in this case $M$ is called productcomplete [9]. Also, every module has a $\operatorname{Prod}(M)$-preenvelope [9]. Then we have the following corollary.

Corollary 6.4. (i) Let $M$ be product-complete $\Sigma$ - $\mathbb{E}$-A-lifting. If $j: Y \rightarrow Z$ is a non-zero epimorphism such that $\operatorname{Ker} j \in \mathcal{A}, Z \in \operatorname{Add}(M), Y \in \operatorname{Cogen}^{\prime}(M)$ and $Y$ satisfies (*), then $Y \in \operatorname{Add}(M)$.

(ii) Let $M$ be $\prod$-E-A-lifting. If $j: Y \rightarrow Z$ is a non-zero epimorphism such that $\operatorname{Ker} j \in \mathcal{A}, Z \in \operatorname{Prod}(M), Y \in \operatorname{Cogen}(M)$ and $Y$ satisfies (*), then $Y \in \operatorname{Prod}(M)$. 
Acknowledgements. The author would like to thank the referee for the useful comments and suggestions.

\section{References}

[1] I. Al-Khazzi, P.F. Smith, Classes of modules with many direct summands, J. Austral. Math. Soc. Ser. A 59 (1995), 8-19.

[2] D.A. Buchsbaum, A note on homology in categories, Ann. Math. 69 (1959), 66-74.

[3] J. Clark, C. Lomp, N. Vanaja, R. Wisbauer, Lifting modules. Supplements and projectivity in module theory, Frontiers in Mathematics, Birkhäuser, Basel, 2006.

[4] S. Crivei, $\Sigma$-extending modules, $\Sigma$-lifting modules, and proper classes, Comm. Algebra 36 (2008), 529-545. .

[5] N.V. Dung, D.V. Huynh, P.F. Smith, R. Wisbauer, Extending modules, Pitman Research Notes in Mathematics Series, 313, Longman Scientific \& Technical, 1994.

[6] T. Koşan, A. Harmancı, Modules supplemented relative to a torsion theory, Turk. J. Math. 28 (2004), 177-184.

[7] A.P. Mishina, L.A. Skornjakov, Abelian groups and modules, Amer. Math. Soc. Transl., Ser. 2, 107, 1976.

[8] K. Oshiro, Lifting modules, extending modules and their applications to QF-rings, Hokkaido Math. J. 13 (1984), 310-338.

[9] J. Rada, M. Saorín, Rings characterized by (pre)envelopes and (pre)covers of their modules, Comm. Algebra 26 (1998), 899-912.

[10] B. Stenström, Rings of quotients, Springer-Verlag, Berlin, 1975.

[11] R. Wisbauer, Foundations of module and ring theory, Gordon \& Breach, Reading, 1991. 